recovered either instantaneously or at most after a few seconds on moistening, those of the latter took 40.50 sec., or often longer.

Müller points out that mosses cease to condense atmospheric moisture when the tension of water vapour is lower in the air than in the cells, so that this method is of significance to the moss only at times when the atmosphere approaches saturation. In the forest, the saturation defieit of the atmosphere increases with the distance from the ground level, and the rate of this increase depends chiefly upon the permeability of the soil and the type of forest (that is, broad-leaved or coniferous). Partly as a result of this, the moss life of the Leucodon and Anomodon type in the broad-leaved forest extends up the trees to a height of $20 \mathrm{~m}$., whilst the Drepanium and Orthodicranum type in the coniferous forest only extends $3-4 \mathrm{~m}$. The heights to which the mosses can extend is evidently controlled to some extent also by properties of the mosses themselves, since only certain species- - the epiphytic species-can extend up the tree more than about $30-50 \mathrm{~cm}$.; below this level one finds some of the species characteristic of the ground flora, for example, Eurhynchium striatum, Pleurozium Schreberi, along with certain Phanerogams, such as Oxalis acetosella and Geranium Robertianum.

The general occurrence of certain epiphytes on broad-leaved and others on coniferous trees might be explained to some extent by the humidity of the type of forest formed by such trees, a factor which is seen to influence the flora of the undergrowth and tree bases. There is, however, something more than this obvious relationship between the true epiphytic mosses and their hosts, for one finds that trees of a particular kind, for example, Pinus sylvestris, have their characteristic epiphytic mosses whether growing in a typical pine association or occurring as an isolated example in an association consisting typically of broad-leaved trees, and vice versa. Further, one finds that within an association, a particular moss may show a preference for a particular kind of tree, for example, Leucodon for Carpinus betulus and Anomodon for Acer platanoides and Fraxinus excelsior-a curious fact which future investigation may show to be associated with the type of bark (fissured or scale) and the consequent difference in the rate at which water flows away.

An unexpected feature that is without any explanation is that, as regards epiphytic moss vegetation, Betula spp. are classed with the conifers-a fact which is further supported by the distribution of epiphytic lichens by Räsenen in Finland.

The connexion between an epiphytic moss and its host cannot be regarded as strict, by any means. Wisniewski points out that, although the types he regards as epiphytic are rarely found in other habitats, there are very few of them which have not been recorded as growing on rock or stone as well. The marked preference for the epiphytic habit is further emphasised by the fact that very few of them are recorded from altitudes or latitudes beyond the tree zone. A study of the literature on moss distribution brings out the fact that the majority of the epiphytic forms-48 per cent-of the Białowieza forest are holoarctic in distribution, extending across Europe to North Asia and China on one side, and to North America on the other. It is curious to find that of the twelve species of trees serving as the commonest hosts for epiphytic mosses in the Bialowieza forest, none occurs in North America, so that evidently the same species of mosses in the latter continent must have transferred to other hosts.

\title{
World Geometry in its Time Relations.
}

PROF. R. C. TOLMAN, of the Norman Bridge Laboratory of Physics, Pasadena, has published, in recent issues of the Proceedings of the U.S. National Academy of Sciences, a series of papers on world geometry in its time relations. The subject is the same as that of recent papers by Lemaitre, de Sitter, and Eddington, namely, the existence of non-static solutions of Einstein's gravitational equations. Prof. Tolman's papers are admirably concrete and free from paradox, and will appeal to those who are attracted by a certain definiteness of point of view.

After discussing the recognised weaknesses of the Einstein line-element (full, static universe) and the de Sitter line-element (empty universe), Prof. Tolman proceeds to determine a line-element on the basis of five assumptions: (1) spatial spherical symmetry; (2) symmetry with respect to past and future time; (3) a criterion of stability; (4) and (5) conditions of isotropy with respect to the volume defined by a system of nebulæ and with respect to the average density of matter. These conditions are shown to determine the form of the line element uniquely save for a certain function of time only, which occurs as a multiplier in the space part of the line element. As a first approximation, Prof. Tolman takes this to be a linear function of the time, and reduces its determination to the ascertaining of the numerical value of a single parameter, which must be a physical constant. This constant can be interpreted in terms of the time-interval between the sending out of two light-impulses by a nebula and the time-interval between their receptions. If these are not equal, the nebula will appear to be in motion in the line of sight, and the lines in its spectrum will be displaced with red or violet. The discrepancy between the two intervals will increase with the distance of the nebula, and Prof. Tolman deduces a proportionality between distance and line-displacement. This is in agreement with the empirically found relation between red-shift and distance for nebulæ, and, using the observed numerical values, the author infers the values of the constant in his formula for the lineelement.

Prof. Tolman then shows that owing to the presence of this time-factor in the line-element, the mass enclosed within a given volume must be changing, and he identifies this change with the transformation of matter into radiation-that is, he identifies the reduction in measured mass with the mass disappearing from the volume in the form of radiation. The value of the time-constant deduced from the observed recession of nebulæ should thus be connected with the rate of evolution of energy per gram by the stars. From the nebular recession, Tolman finds $k=5 \cdot 1 \times 10^{-10}$ (years $)^{-1}$, whilst the values of $k$ deduced from the observed masses and luminosities of the stars as tabulated by him vary from $1.7 \times 10^{-10}$ down to $2.3 \times 10^{-16}$.

In a later paper of the series Prof. Tolman shows that the addition of higher terms to his linear approximation for his undetermined function of time may seriously modify the values of $k$ deduced from the observed rate of annihilation of matter, and concludes that the discrepancy is not fatal. One is naturally tempted to make the criticism that the rate of annihilation of matter must be governed by the physics of the energy-generating process in stars, and so is surely a different physical phenomenon from the recession of the nebulæ, implied by such considerations as those of stability and symmetry in the universe. This, however, is not incompatible with the view that the transformation of matter is the 
primary process, and that this by conditioning the mass-change of the universe is the 'cause' of the world-geometry which predicts the nebular recession.

The same confusion between perhaps diverse physical phenomena is seen in another paper of the series, where Prof. Tolman gives an actual formula for the luminosity of a nebula in terms of its distance and red-shift. This appears to assume some common property in the nebulæ, but it is obvious that nebulæ (like stars) could have intrinsically different luminosities for the same distance. The whole discussion is, however, most stimulating, and will certainly help to provoke more accurate and extensive observations of the most distant nebulæ.

\section{Thinning Operations in Forestry.}

GOR a century or two it has probably been the desire and aim of the scientific forester to endeavour to bring the work of thinning a wood, at various ages in its development, within the circumscribed limits of a definition. There are many experienced foresters, and probably some of the most expert in this part of the forester's work, who maintain that any definition of the work involved or the laying down of any hard-andfast rules is impracticable-if for no other reason than that in any wood the soil and other factors vary from place to place, with a consequent variability in growth. It follows from this state of affairs that the thinning operations must, if properly carried out, be based upon the condition of the individual trees and their crowns at any spot; and this condition will be a variable quantity. Greater regularity may be found in wellmanaged coniferous woods, and even in young wellgrown broad-leaved crops; but so far as the forests of the British Empire are concerned, such conditions are only exceptionally present.

Some of these points are recognised in the brochure entitled "Classifications of "Thinnings" (Indian For. Records, vol. 15, pt. 1, 1930. Govt. of India, Calcutta Press). One of the reasons for the persistent effort to evolve some method of classifying thinnings has been the desire to have some rules or rule-of-thumb methods of dealing with certain types of crops which would be simple enough in practice to enable their carrying out by the subordinate staff. In view of the enormous and increasing amount of this type of work which faces the numerically small staffs of the Empire Forestry Departments, it will prove almost a necessity to place a portion of the thinning work in the hands of the non-gazetted grades. But it has Iong been accepted that the best amongst thinning experts are born with the gift; that such are to be found in the lower ranks as well as in the upper. In either case the young forester requires to be trained by the senior who is an expert in practice, and not by any rule of thumb such as the brochure here under review attempts to prescribe.

This is not to say that the attempted classification is not of use to those who have a first-hand practical acquaintance with thinning work, either in one type of crop (mixed or pure), or in varying crops managed under different sylvicultural systems. But a very considerable amount of experience would be required before this attempt at classifying thinnings could be translated into practice : even then a wide and varying meaning can be given by different performers to the definitions given under the subdivisions on "Intensity of Thinnings". These subdivisions speak for themselves (for the definitions the pamphlet must be consulted). I. Ordinary Thinning: (1) Light thinning (A grade); (2) moderate thinning (B grade); heavy thinning ( $\mathrm{C}$ grade); very heavy thinning (D grade). II. Crown Thinnings : (1) Light crown thin. ning (L.C.grade); heavy crown thinning (H.C. grade). To make use of this brochure with intelligence, and without danger to the crops being treated, the forester requires to possess a clear knowledge of the relative significance of the words and phrases used in the definitions, combined with a very considerable previous practice in the carrying out of one of the most important and interesting of his duties.

\section{Curious Markings on Stones in Scotland.}

T THE Glasgow Herald of Sept. 17 contains an article 1 by Mr. L. MacLellan Mann describing the mark. ings on some stones at Langside and Cleuch, near Glasgow. The markings on the two stones are nearly alike, consisting of series of rings, arcs, and cup-like depressions. Mr. Mann claims that these have astronomical significance; some of the groups of cups are shown to resemble the Sickle in Leo and (more doubtfully) a star-group in Scorpio. He further claims that he can identify records of ancient eclipses; it would, however, need a fuller explanation of his method to induce astronomers to accept his claims in full. $\mathrm{He}$ states that he identified the date of a recorded eclipse as B.c. 2983 Mar. 28* Gregorian reckoning from the stone itself, and afterwards found by consulting astronomers in Berlin that there was a total eclipse on that date, the track of totality passing over or near Glasgow. The writer of the present note has verified this latter fact independently, making use of the newmoon tables by the late $\mathrm{C}$. Schoch that are contained in "The Venus Tablets of Ammizaduga" (Langdon and Fotheringham, 1928). These tables make use of the latest values of the solar and lunar accelerations; but there is of necessity a considerable margin of uncertainty in computing the tracks of very early eclipses.

This eclipse affords a good illustration of the use of M. Oppert's long eclipse cycle of 1805 years; the name

* Mr. Mann gives Mar. 27, but 28 appears to be correct.

No. 3184, VoL. 126]
' megalosaros' has been suggested for it; it is about. a hundred times as long as the 'saros', and shares with it the useful property that the parallaxes of sun and moon nearly repeat themselves. The following table gives the tracks of the three successors of this eclipse; they are from Oppolzer's "Canon" and Schrader's sequel to it :

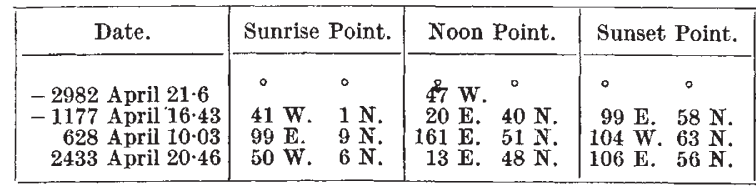

The first three dates are by the Julian calendar, the fourth by the Gregorian one.

It will be seen that the cycle enables us to make a close approximation to the latitude of the eclipse track; the longitude offers greater difficulty owing to the large effect of the secular acceleration in such a long period. Oppolzer's older eclipses themselves require a considerable shift in longitude to reduce to Schoch's values of the accelerations.

Mr. Mann claims to have found similar records of still older eclipses; thus he refers to one in New Mexico of the date B.C. 3457 Sept. 5. It would, how, ever, be well for him to make the full case for the 2983 eclipse accessible to astronomers before asking them to consider these more remote ones. 